\title{
The m6A reader IGF2BP3 Facilitates Gastric Cancer Progression Through Activating EMT via Upregulating MYC mRNA Stability
}

\section{Yiyi Ren (D220193557@seu.edu.cn )}

Southeast University - Dingjiaqiao Campus https://orcid.org/0000-0002-6602-8343

\section{Dandan Du}

Southeast University - Dingjiaqiao Campus

Tong Liu

Southeast University - Dingjiaqiao Campus

\section{Chuntao Wang}

Jiangsu Vocational College of Medicine

\section{Qinyu Yan}

Southeast University - Dingjiaqiao Campus

\section{Chengyun Li}

Lanzhou University

\section{Xinghua Wang}

Jiangsu Vocational College of Medicine

\section{Sheng Yang}

Southeast University - Dingjiaqiao Campus

\section{Lu Kong}

Southeast University - Dingjiaqiao Campus

\section{Geyu Liang}

Southeast University - Dingjiaqiao Campus https://orcid.org/0000-0002-6962-6488

\section{Research Article}

Keywords: m6A reader, IGF2BP3, MYC, EMT, gastric cancer.

Posted Date: December 17th, 2021

DOI: https://doi.org/10.21203/rs.3.rs-1095901/v1

License: (c) (i) This work is licensed under a Creative Commons Attribution 4.0 International License.

Read Full License 


\section{Abstract}

Background: N6-methyladenosine (m6A) RNA methylation plays an important biological role in cancer progression. Even so, the role of m6A modification in gastric cancer (GC) still needs further research.

Methods: Firstly, based on the bioinformatics databases and human GC tissues analysis. Secondly, the IGF2BP3 expression in GC cells was measured by the quantificational real-time polymerase chain reaction and Western Blot. Then, the IGF2BP3 knockdown stable cells model was successfully constructed with the specific lentivirus-mediated short-hairpin RNA to explore the functions and mechanism of IGF2BP3 in GC. Next, the functions of IGF2BP3 on the cell phenotypes, including proliferation, invasion, migration, and Epithelial-mesenchymal transition process were clarified by the Cell Counting Kit-8, transwell, and WB experiments. Subsequently, RNA Immunoprecipitation analysis and mRNA stability experiments were used to verify the relationship between IGF2BP3 and MYC. Finally, in the rescue experiment, MYC was overexpressed and transfected into IGF2BP3 knockdown cells to further detect the influences on the cell phenotypes and the EMT process.

Results: IGF2BP3 was up-regulated in GC. Meanwhile, IGF2BP3 had diagnostic and prognosis values for GC. Functionally, knockdown IGF2BP3 repressed gastric cancer cells proliferation, migration invasion and EMT process. Mechanically, IGF2BP3 activated the EMT process by improving the expression of MYC via combining with MYC mRNA and promoting its stability.

Conclusions: Taken together, IGF2BP3 could activate the EMT process via increasing the MYC mRNA stability and expression to promote GC development, which provided insight into promising early diagnose and treatment for gastric cancer.

\section{Introduction}

Gastric cancer (GC) is a life-threatening malignancy [1]. The latest global cancer statistics reported that GC ranks fifth and fourth of all malignancies for morbidity and mortality in 2020 [2]. Unfortunately, people with early-stage GC often have no symptoms, while most GC patients are always in the advanced stage with malignant proliferation and metastasis at the time of diagnosis, resulting in a poor prognosis [3]. Thus, founding novel biomarkers for diagnosis and therapeutic targets for treatment of GC is an urgent need.

As one of the critical "epitranscriptomics" processes, the N6-methyladenosine (m6A) modification has attracted wide attention [4]. The m6A modification is a dynamic and reversible process, which is installed by m6A writers (namely m6A methyltransferases), removed by m6A erasers demethylases (namely m6A demethylases), and recognized by readers (namely m6A-binding proteins) [5]. Accumulating evidences indicated that the disorder of m6A methylation closely associates with the incidence and development of various cancers $[6,7]$. Furthermore, it speculated that the m6A readers determined the fate of m6Amodified mRNAs, to be specific, m6A-modified mRNAs is recognized by different $m 6 \mathrm{~A}$ readers may lead to divergent biological functions [8]. At present, although the importance of m6A binding protein in GC 
cancer has gradually gained wide attention [9-12], the underlying role and epigenetic regulation of m6A reader in gastric cancer still need to be explored.

In this study, we uncovered that insulin-like growth factor 2 messenger RNA-binding protein 3 (IGF2BP3) expression level was upregulated in GC and related to poor gastric cancer progression. We further revealed the crucial functions of IGF2BP3 in promoting GC progression in vitro via strengthening MYC mRNA stability. Our work uncovered the role and the novel mechanisms underlying GC tumorigenesis, which may provide a novel diagnosis predictor, a prognostic biomarker for patients, and a promising new therapeutic target of GC patients.

\section{Materials And Methods}

\section{Clinical Specimens}

35 pairs of GC patient specimens were used in the study. Patients with the following criteria were excluded: with any antitumor treatment; with other cancers diagnoses. The research was approved by the Ethics Committee for Clinical Research of the University hospital affiliated with Southeast University and the informed consent was acquired from every patient.

\section{GC datasets}

The TCGA-GC of $375 \mathrm{GC}$ samples and 32 normal samples were acquired from the Cancer Genome Atlas (TCGA) (https://gdc-portal.nci.nih.gov/). The m6A readers expression of $5 \mathrm{GC}$ datasets were obtained from the Gene Expression Omnibus (GEO) database (http://www.ncbi.nlm.nih.gov/geo/), including GSE66229 (South Korea, 300 cases of GC, 100 normal cases), GSE54129 (China, 111 cases of GC, 21 normal cases), GSE29998 (Singapore, 50 cases of GC, 49 normal cases), GSE64951 (USA, 63 cases of GC, 31 normal cases) and GSE96668 (United Kingdom, 49 cases of GC, 11 normal cases).

\section{Cells}

Human gastric cancer cells HGC-27, SGC-7901, BGC-823, and the human gastric mucosa epithelial cell line GES-1 were purchased from the Cellcook Biotech Co., Ltd., Guangzhou, China.

\section{Cell culture}

GES-1, BGC-823 and SGC-7901 cells were cultured in RPMI-1640 medium (Gibco, USA), while HGC-27 cells were cultured in MEM medium (Gibco, USA). All media were adding $10 \%$ fetal bovine serum (FBS) (Gibco, USA), penicillin (100 U/mL) and streptomycin $(100 \mu \mathrm{g} / \mathrm{mL})$ (Invitrogen, USA), which all cultured in the stable environment with $5 \% \mathrm{CO} 2$ at $37^{\circ} \mathrm{C}$.

\section{Cell transfection}

The short hairpin RNA (shRNA)-mediated knockdown of IGF2BP3 was performed to interfere with the expression of IGF2BP3. IGF2BP3 shRNA (shIGF2BP3, abbreviated, shl3) and shRNA control (shNC) were 
purchased by HANBIO (Shanghai, China). The shl3 and shNC were transfected into HGC-27-shl3 cells. The IGF2BP3 knockdown and control HGC-27 cells are named HGC27-shl3 and HGC27-shNC, respectively. MYC overexpression plasmid and vector control were synthesized by HANYANG (Suzhou, China). They were transfected into HGC27-shl3, HGC27-shNC cells. The MYC overexpression, control HGC27-shl3, blank control HGC27-shNC cells are named HGC-shl3 + MYC, HGC-shl3 + vector, HGC-shNC + vector, respectively. All the experiment procedures were operated according to the manufacturer's instructions from the corresponding company.

\section{RNA isolation and the quantificational real-time polymerase chain reaction (qRT-PCR)}

Total RNAs were extracted by using TRIzol reagent (Genstar, China), and then reverse-transcribed to complementary DNA (cDNA) by using PrimeScript RT Reagent (Genstar, China). RNA expression levels were detected by qRT-PCR through using the SYBR Premix Ex Taq (Genstar, China) and analyzed by $2-\Delta \Delta^{\mathrm{Ct}}$ method, all the results were normalized by $\beta$-actin. The primer sequences were used in this study are listed in the Table 1.

Table 1 The sequence of primers

\begin{tabular}{|c|c|c|}
\hline Gene & Primers & sequence \\
\hline \multirow[t]{2}{*}{ IGF2BP1 } & Forward primer & 5囚-CAAAGGAGCCGGAAAATTCAAAT-3》 \\
\hline & Reverse primer & 5囚-CGTCTCACTCTCGGTGTTCA-3囚 \\
\hline \multirow[t]{2}{*}{ IGF2BP2 } & Forward primer & 5囚-AGTGGAATTGCATGGGAAAATCA-3区 \\
\hline & Reverse primer & 5】-CAACGGCGGTTTCTGTGTC-3】 \\
\hline \multirow[t]{2}{*}{ IGF2BP3 } & Forward primer & 5囚-ACGAAATATCCCGCCTCATTTAC-3囚 \\
\hline & Reverse primer & 5囚-GCAGTTTCCGAGTCAGTGTTCA-3】 \\
\hline \multirow[t]{2}{*}{ YTHDF1 } & Forward primer & 5囚-ATGTCGGCCACCAGCGTGGACA -3囚 \\
\hline & Reverse primer & 5囚-TCATTGTTTGTTTCGACTCTGC -3囚 \\
\hline \multirow[t]{2}{*}{ YTHDF2 } & Forward primer & 5囚-CCTTAGGTGGAGCCATGATTG-3囚 \\
\hline & Reverse primer & 5囚-TCTGTGCTACCCAACTTCAGT-3囚 \\
\hline \multirow[t]{2}{*}{ YTHDF3 } & Forward primer & 5囚-TGACAACAAACCGGTTACCA-3】 \\
\hline & Reverse primer & 5囚-TGTTTCTATTTCTCTCCCTACGC-3》 \\
\hline \multirow[t]{2}{*}{ MYC } & Forward primer & 5囚-CGAACACACAACGTCTTGGAGC-3》 \\
\hline & Reverse primer & 5囚-CTGCTTGGACGGACAGGATG-3】 \\
\hline \multirow[t]{2}{*}{$\beta$-actin } & Forward primer & 5囚-TCTCCCAAGTCCACACAGG-3囚 \\
\hline & Reverse primer & 5囚-GGCACGAAGGCTCATCA-3囚 \\
\hline
\end{tabular}




\section{Western blot (WB)}

The process of the WB experiment was operated as described in the previous research [13]. The primary antibodies against IGF2BP3, MYC, Snail, N-cadherin, vimentin, mouse-anti- $\beta$-actin, Tubulin and the goat anti-rabbit/mouse horseradish peroxidase-conjugated secondary antibody (HRP) (goat-anti-rabbit) (goatanti-mouse) were purchased from Cell Signaling Technology (CST, USA). The band densities of specific proteins were quantified by ImageJ software. The proteins were normalized to $\beta$-actin or Tubulin.

\section{Cell Proliferation Assay}

Cells (3000 cells/well) were seeded in the 96-well plate in 6 replicates, and incubated for $12 \mathrm{~h}, 24 \mathrm{~h}, 48 \mathrm{~h}$ and $72 \mathrm{~h}$, respectively. After that, the proliferation viability of cells was measured by the CCK8 kit (malunion, China). The Optical density (OD) was detected at $450 \mathrm{~nm}$ by a microplate reader.

\section{Transwell Assays}

The migration and invasion assays were operated with a 3422 Transwell chamber (Corning, USA) which were coated without the Matrigel mix (BD Biosciences, USA) (migration assay) or with the Matrigel mix (invasion assay). After the cells incubated for $24 \mathrm{~h}$, we used $4 \%$ paraformaldehyde to fix the cells located on the lower surface of chamber for 15 minutes. And then, the cells were stained with $0.1 \%$ crystal violet dye for 15 minutes. After that, the stained cells were photographed and the number of stained cells were calculated in five randomly selected fields.

\section{RNA binding protein immunoprecipitation (RIP)}

RNA immunoprecipitation experiments were operated by using RNA Immunoprecipitation (RIP) Kit (Merck Millipore, USA), and the operating steps according to the manufacturer's instructions. The analysis of coprecipitated RNAs were detected by qRT-PCR.

\section{mRNA stability assays}

The stability of MYC mRNA was determined by applying actinomycin D (ActD; Final concentration: 2.5 $\mu \mathrm{g} / \mathrm{mL}$; Selleck, USA) mRNA stability assays in HGC27-shl3, HGC27-NC cells. At the indicated time point, mRNA degradation was analyzed by quantitative RT-PCR as described above, normalized to $\beta$-actin. The half-life (t1/2) of mRNA was calculated.

\section{Immunohistochemistry (IHC)}

Immunohistochemistry was operated on the paraffin sections. And they were dewaxed, dehydrated and rehydrated ordinally. After washing with phosphate-buffered saline (PBS), the paraffin sections were adding citrate buffer and then performed with high pressure repairing antigen. Next, the paraffin sections were blocked endogenous peroxidase activity by adding $0.3 \%$ hydrogen peroxide. Then, washing with PBS 3 times. After that, the paraffin sections were blocked by adding 10\% goat serum. Then the paraffin sections were incubated with the corresponding primary antibody at $4^{\circ} \mathrm{C}$ for $14-16$ hours. Next, the 
paraffin sections were washed with PBS 3 times and were added with the anti-rabbit IgG-horseradish peroxidase (HRP) for 10 minutes, at room temperature. Then, the paraffin sections were washed with PBS 3 times again. After that, the paraffin sections were visualized by using 3,3'-diaminobenzidine

tetrahydrochloride (DAB). The reaction was terminated when dyeing was appropriate. Then, washing with PBS 3 times again. After the paraffin sections were washed with PBS 3 times, the paraffin sections were counterstained with hematoxylin, washed with running water, dehydrated, and mounted with neutral gum.

\section{Statistical analysis}

The SPSS 22.0 software was used to analyze the data. The mean \pm standard deviation (Mean \pm SD) was applied to represent the data. The $2^{-\triangle \Delta \mathrm{Ct}}$ method was used to analyze the qRT-PCR results. The receiver operating characteristic (ROC) curve was applied to test the diagnostic ability, and the Kaplan-Meier (K-M) curves were used to evaluate the prognostic prediction. The difference of m6A readers expression based on the TCGA database was analyzed by Wilcox analysis via using $\mathrm{R}$ version 3.5.1. The comparison of data was analyzed by using variance analysis, chi-square test, or non-parametric test when appropriate.

The correlation between the target genes was explored by Pearson correlation analysis. The test level was $a=0.05$ (two-sided), $P$-value $<0.05$ represented that the differences were statistically significant.

\section{Results}

\section{IGF2BP3 was up-regulated in GC and related with the diagnosis and prognosis of GC}

To discover the potential target $\mathrm{m} 6 \mathrm{~A}$ reader in $\mathrm{GC}$, we analyzed $\mathrm{m} 6 \mathrm{~A}$ readers expression in $\mathrm{GC}$ at first, the analysis of the TCGA database showed that IGF2BP3, IGF2BP1, IGF2BP2 and YTHDF1 were increased with fold change ( $|\mathrm{FC}|>2$ and $P<0.05)$ in $\mathrm{GC}$ tissues (Fig. 1A). The analysis of five GEO datasets indicated that IGF2BP3 $(|\mathrm{FC}|>2$ and $P<0.002)$ and YTHDF3 $(|\mathrm{FC}|>2$ and $P<0.001)$ were up-regulated, while IGF2BP2 and YTHDC1 were downregulated( $|\mathrm{FC}|>2$ and $P<0.001$ in gastric cancer tissues (Fig. 1B). In keeping with the databases mentioned above, we further obtained that compared with the normal counterpart, IGF2BP3 expression was distinctively up-regulated ( $F C=7.73, P=0.046)$ in human $\mathrm{GC}$ tissues. (Fig. 1C).

Then, based on the TCGA database and human GC tissues, we investigated the clinicopathological significance of ten m6A readers in GC. First, the diagnostic value of m6A RNA readers in GC was investigated. The ROC analysis results based on human GC tissues (Fig. 1D) and the TCGA database (Fig. 1E) consistently demonstrated that IGF2BP3 and YTHDF1 had diagnostic values for GC. Next, the prognostic role of m6A readers in $\mathrm{GC}$ was explored (Fig. 1F). The Kaplan-Meier plotter analysis based on the TCGA database revealed that the GC patients with the higher expression level of IGF2BP3, YTHDF1, YTHDF2, YTHDC1, and HNRNPA2B1 had a significantly worse prognosis, while the GC patients with the higher expression level of HNRNPC and YTHDF2 groups had longer survival times. In keeping with the databases mentioned above, we further obtained that compared with the normal counterpart, IGF2BP3 expression was distinctively up-regulated ( $\mathrm{FC}=7.73, P=0.046)$ in human $\mathrm{GC}$ tissues. 
Above all, with the described results of TCGA, GEO databases and human GC tissues, we summarized that IGF2BP3 expression is remarkably up-regulated in GC. Meanwhile, IGF2BP3 had diagnostic and prognostic values of GC patients, which might act as an oncogene in GC tumors. Therefore, IGF2BP3 was selected as the target molecule for the subsequent research.

In addition, in order to further clarify the mechanism of IGF2BP3 in GC, based on the m6A2 Target database (http://m6a2target.canceromics.org) to preliminarily predict the downstream target mRNA of IGF2BP3, we found that MYC might be the downstream target gene of IGF2BP3. Moreover, based on the analysis of the TCGA database and the GES96668 dataset, we discovered that there was a positive correlation analysis of the correlation between IGF2BP3 and MYC mRNA expression levels $(P<0.05)$ (Fig. 1G). In addition, the IGF2BP3, MYC, EMT markers protein expression in human gastric cancer and para-carcinoma tissues was measured by Immunohistochemistry (IHC). The IHC assay manifested that compared with para-carcinoma tissue, IGF2BP3, MYC and EMT markers ( $\mathrm{N}$-cadherin, Vimentin, a-SMA and Snail) protein expression all increased in gastric cancer tissue (Fig. $1 \mathrm{H}$ ). These results prompted that MYC might be a potential target downstream gene for IGF2BP3, and the EMT process plays a vital role in the GC development.

\section{IGF2BP3 was up-regulated in GC cells.}

To validate the expression of IGF2BP3 in GC, the IGF2BP3 expression was detected in GC cell lines by applying qRT-PCR and WB. The results showed that IGF2BP3 expression was generally up-regulated in three types of GC cells (SGC-7901, BGC-823 and HGC-27) (Fig. 2A, B), compared with GES-1 cells. What's more, IGF2BP3 expression in HGC-27 cells was the highest. Therefore, the HGC-27 cell was selected for further biological function studies and mechanism studies.

\section{IGF2BP3 promoted the proliferation, migration, invasion and the EMT process of HGC-27 cells in vitro.}

To investigate the possible functions of IGF2BP3 in gastric cancer cells, we established the stable IGF2BP3-knockdown HGC-27 cells with transfection the specific lentivirus-mediated short-hairpin RNA (shIGF2BP3, abbreviated as shl3: IGF2BP3 knockdown group and shNC: control group) (Figure2C, D), respectively. qRT-PCR and WB consistently indicated that the IGF2BP3 mRNA expression was reduced in the HGC-27-shl3 cells, compared with the HGC-27-shNC cells (Fig. 2E, F). CCK-8 assays demonstrated that knockdown IGF2BP3 distinctly inhibited the proliferation ability of HGC-27 cells (Fig. 2G). The transwell assays also indicated that knockdown IGF2BP3 distinctly significantly repressed the migration and invasion ability of HGC-27 cells (Fig. 2H-2I). Additionally, the EMT markers protein expression was assessed by WB to further clarify the functions of IGF2BP3 on the regulation of GC cells progression. The WB indicated that EMT markers (N-cadherin, Vimentin, a-SMA and Snail) in the IGF2BP3 knockdown group were down-regulated in HGC-27 cells $(P<0.01)$ (Fig. 2J). Above all, IGF2BP3 could facilitate gastric cancer cells proliferation, invasion, migration and EMT process.

IGF2BP3 could combine with MYC mRNA to increase the expression and stability of MYC mRNA 
To understand the underlying mechanism of IGF2BP3 exerted tumor-promoting effects in GC. Based on the preliminary exploration of m6A2Target, TCGA database and IHC assay analysis, we chose MYC as the downstream target gene to further clarify the correlation between IGF2BP3 and downstream target MYC. The MYC expression level after transfection with shIGF2BP3 and shNC in HGC-27 cells was measured by qRT-PCR and WB experiments. The results demonstrated that knockdown IGF2BP3 significantly inhibited the MYC mRNA level (Fig. 3A) and the protein level in HGC-27 cells (Fig. 3B). To verify whether the MYC expression was influenced by IGF2BP3 combination with MYC, we performed the RIP assay and confirmed the binding. The qRT-PCR analysis indicated that knockdown IGF2BP3 repressed the combination of IGF2BP3 protein binding with MYC mRNA, compared to immunoglobulin G (IgG) in HGC-27 (Fig. 3C). Meanwhile, ActD was applied to explore whether IGF2BP3 can affect the MYC mRNA stability in HGC-27. The results suggested that knockdown IGF2BP3 significantly reduces MYC mRNA stability and shortens MYC mRNA half-life in HGC-27 (Fig. 3D). Therefore, the regulatory role of MYC in IGF2BP3 knockdown HGC-27 cells was investigated through transfecting with MYC overexpression plasmids (Fig. 3E) into HGC-27-shl3 cells. The Western blot results presented that MYC overexpression effectively reverses the inhibition of MYC protein expression level in IGF2BP3 knockdown HGC-27 (Fig. 3F). In summary, these results strongly suggested that IGF2BP3 can increase the MYC mRNA and protein expression via recognizing and combining with MYC mRNA to increase the MYC mRNA stability in GC cells.

\section{Up-regulated MYC effectively restored IGF2BP3 knockdown-induced inhibition of GC Cell Progression}

To further investigate whether MYC participated in IGF2BP3-induced effects on GC cell progression, we conducted the rescue assays. In the rescue experiment, MYC was overexpressed and transfected into IGF2BP3 knockdown HGC-27 to detect the influence of the recovery MYC overexpression on the cellular phenotypes and the EMT process. The results presented that overexpression of MYC could restore the proliferation (Fig. 4A), invasion (Fig. 4B), migration ability (Fig. 4B) and EMT process (Fig. 4C) of GC cells

tumor progression. In a word, IGF2BP3 activated the EMT process by recognizing and binding MYC and thus increasing the mRNA stability and expression of MYC, resulting in facilitating proliferation, invasion, migration ability of GC cells.

\section{Discussion}

It has been reported that the tumor cells' epi-transcriptome was indeed disturbed by abnormal m6A regulator (writers, erasers and readers) playing important roles in GC development [14]. The transcriptome-wide RNA m6A landscape was specially recognized by various m6A readers, which can ultimately control the destiny of many vital transcripts, influencing distinct aspects of cancer malignancy biology [15].

In the present research, we systematically analyzed ten $\mathrm{m} 6 \mathrm{~A}$ readers and discovered that the m6A reader gene IGF2BP3 might be a critical prognostic danger factor of gastric cancer. More importantly, we proved 
that IGF2BP3 activated the EMT process by enhancing the combination with MYC mRNA and improving MYC mRNA stability to increase the MYC expression, accelerating the GC cells development in vitro.

At first, to identify the critical m6A readers in GC, we analyzed the expression level of ten m6A readers (IGF2BP1/2/3, YTHDF1/2/3, YTHDC1/2, HNRNPC and HNRNPA2B1) based on the TCGA, GEO databases and human GC tissues, we recognized that IGF2BP3 is the most frequently overexpressed m6Aassociated gene in GC, consistent with the previous study. In addition, by integrating the Kaplan-Meier plotter and the ROC analysis, we discovered that IGF2BP3 overexpression correlates with the early diagnostic and prognosis, consistent with previous research [16-18], suggesting IGF2BP3 might be a potential candidate biomarker for GC.

Next, given that IGF2BP3 has been involved in the human tumor progression [19-23]. We successfully constructed the stably knockdown IGF2BP3 in GC HGC-27 cell lines to evaluate the influence of IGF2BP3 on the GC cell phenotypes. The results indicated that knockdown IGF2BP3 inhibited the HGC-27 cells proliferation, migration and invasion in vitro, keeping with Zhang $\mathrm{J}$ et al. demonstrated that IGF2BP3 promoted GC cell proliferation in HGC-27 GC cell lines [24]. Likewise, Zhou Y et al. reported that knockdown IGF2BP3 could inhibit cell proliferation and invasion ability in MKN28 GC cells, in good agreement with our results [25]. In summary, these results further elucidated that IGF2BP3 plays an important role in promoting GC cells proliferation, invasion and migration, implying that IGF2BP3 could lead to poor prognosis by accelerating tumor growth of GC.

In addition, to explore the mechanisms of IGF2BP3 in GC in depth. By integrating results of the previous researches, the m6A2Target databases prediction, the TCGA databases, the GEO databases and the immunohistochemical (IHC) assays, we concluded that there was a positive correlation analysis of the correlation between IGF2BP3 and MYC mRNA expression levels, which indicated that IGF2BP3 may promote the progression of GC through upregulating MYC mRNA. It was reported that the MYC played a key role in cell proliferation, migration and homeostasis of GC development [26-28], and MYC was also often elevated in GC and related to poor survival and clinical late-stage [29-31]. What is more, through the RIP-qPCR validation method and mRNA stability experiment, we further confirmed that IGF2BP3 could promote MYC expression by direct combination with MYC mRNA to enhance the MYC mRNA stability in GC cells, consistent with the previous research [32]. This is the first validation of direct binding between IGF2BP3 and MYC with a positive correlation in GC cells, to the best of our knowledge. Furthermore, the rescue experiment was applied to explore whether the IGF2BP3-MYC axis can regulate gastric cells' proliferation, invasion and metastasis. In conclusion, the above results revealed that MYC overexpression could restore the suppressing function of GC cells proliferation, migration and invasion induced by IGF2BP3 knockdown. Prompting that IGF2BP3 could facilitate GC development by enhancing the stability and expression of MYC.

It's worth noting that earlier research had reported that epithelial-mesenchymal transition (EMT) was the primary malignant progression mechanism leading to cell metastasis and invasion in GC [33, 34]. Moreover, recent researches have indicated that m6A modification can influence the EMT process in GC 
cells [35]. Cheng et al. indicated that IGF2BP3-mediated m6A modification on ZBTB4 was participated in CS-induced EMT and lung cancer through EZH2 [36]. However, few studies were about whether IGF2BP3 could activate EMT processes in GC cells. In this study, immunohistochemical results displayed that IGF2BP3 expression was positively related to the EMT markers (N-cadherin, Vimentin, a-SMA and Snail) expression. In addition, we discovered that knockdown IGF2BP3 inhibited the EMT markers protein expression levels in HGC-27 cells. The result was the same as reported in breast cancer [37]. The above results suggested that IGF2BP3 could activate the EMT process in GC cells. Notably, it was reported that MYC could activate the EMT process to facilitate proliferation, invasion and migration [38]. Furthermore, through the rescue experiment, we revealed that MYC overexpression could restore the EMT process of GC. Summing up, all the results manifested that IGF2BP3 activated the EMT process to accelerate the GC cells proliferation, migration and invasion via improving MYC expression.

In conclusion, based on the comprehensive analysis of m6A readers expression of GC population, we discovered that $\mathrm{m6A}$ reader IGF2BP3 was significantly overexpressed in gastric cancer and related to the diagnostic and prognosis for GC patients. Functionally, IGF2BP3 could promote the GC cells proliferation, migration and invasion ability in vitro. Mechanistically, we demonstrated that IGF2BP3 could strengthen MYC mRNA stability through identifying and combining MYC, result in activating the EMT process, which can play a carcinogenic role in GC progression.

\section{Conclusions}

This study innovatively validated that the IGF2BP3-MYC axis played a vital role in activating the EMT process in GC development. In addition, our researches strongly prompted that IGF2BP3 might be a potential biomarker of early diagnosis and prognostic for GC treatment. Which further revealed the regulatory mechanism of GC development and provided a novel target for gastric cancer treatment.

\section{Abbreviations}

Table 2 List of abbreviations 


\begin{tabular}{ll} 
Abbreviations & Full name \\
\hline ActD & Actinomycin D \\
\hline EMT & Epithelial-mesenchymal transition \\
\hline FC & Fold change \\
\hline GC & Gastric cancer \\
\hline IGF2BP3 & Insulin-like growth factor 2 messenger RNA-binding protein 3 \\
\hline m6A & N6-methyladenosine \\
\hline PBS & Phosphate-buffered saline \\
\hline RIP & RNA binding protein immunoprecipitation \\
\hline qRT-PCR & the quantificational real-time polymerase chain reaction \\
\hline TCGA & The Cancer Genome Atlas \\
\hline WB & Western blot \\
\hline
\end{tabular}

\section{Declarations}

\section{Acknowledgements}

We are grateful to all of the reviewers for their comments.

\section{Authors' contributions}

Thank all the people who participated in this study. Yiyi Ren, Dandan Du, Tong Liu selected the research topic and designed the study. Chengyun Li and Xinghua Wang collated the data. Qingyu Yan, Chuntao Wang and Sheng Yang validated and curated the data. Yiyi Ren carried out data analyses and produced the initial draft of the manuscript. Lu Kong and Geyu Liang conducted the guidance of the process of the topic. All authors read and approved the final manuscript.

\section{Funding}

This work was supported by the National Natural Science Foundation of China (81972998), the Fundamental Research Funds for the Central Universities (Izujbky-2020-54), the Qinglan Project in Jiangsu Province (2019) and the High-end Training Project Funding for Teachers' Professional Leaders in Higher Vocational Colleges, Jiangsu Province (2021 GRFX030).

\section{Availability of data and materials}

The datasets used or analyzed during the current study are available from the corresponding author on reasonable request. 


\section{Ethics approval and consent to participate}

The research was approved by the Ethics Committee for Clinical Research of the University hospital affiliated with Southeast University and the informed consent was acquired from every patient.

\section{Consent for publication}

Not applicable.

\section{Competing interests}

There was no conflict of interest.

\section{Author details}

${ }^{1}$ Key Laboratory of Environmental Medicine Engineering, Ministry of Education of China, School of Public Health, Southeast University, Nanjing, 210096, China. ${ }^{2}$ Jiangsu Vocational College of Medicine, Yancheng, 224000, Jiangsu, China. ${ }^{3}$ Department of Toxicology, School of Public Health, Lanzhou University, Lanzhou, 730000, China. ${ }^{4}$ Department of Gastrointestinal Surgery, Gansu Wuwei Cancer Hospital, Wuwei, Gansu 733000, China

\section{References}

1. Cui X, Qin T, Zhao Z, Yang G, Sanches JGP, Zhang Q, Fan S, Cao L, Hu X: Pentraxin-3 inhibits milky spots metastasis of gastric cancer by inhibiting M2 macrophage polarization. J Cancer 2021, 12(15):4686-4697.

2. Sung H, Ferlay J, Siegel RL, Laversanne M, Soerjomataram I, Jemal A, Bray F: Global Cancer Statistics 2020: GLOBOCAN Estimates of Incidence and Mortality Worldwide for 36 Cancers in 185 Countries. CA Cancer J Clin 2021, 71(3):209-249.

3. Chen D, Xu L, Li X, Chu Y, Jiang M, Xu B, Zhao M, Wang W, Wang H, Kang H et al: Enah overexpression is correlated with poor survival and aggressive phenotype in gastric cancer. Cell Death Dis 2018, 9(10):998.

4. Nombela P, Miguel-López B, Blanco S: The role of $\mathrm{m}(6) \mathrm{A}, \mathrm{m}(5) \mathrm{C}$ and $\Psi$ RNA modifications in cancer: Novel therapeutic opportunities. Mol Cancer 2021, 20(1):18.

5. Tan F, Zhao M, Xiong F, Wang Y, Zhang S, Gong Z, Li X, He Y, Shi L, Wang F et al: N6methyladenosine-dependent signalling in cancer progression and insights into cancer therapies. $J$ Exp Clin Cancer Res 2021, 40(1):146.

6. Wang T, Kong S, Tao M, Ju S: The potential role of RNA N6-methyladenosine in Cancer progression. Mol Cancer 2020, 19(1):88.

7. Sun T, Wu R, Ming L: The role of m6A RNA methylation in cancer. Biomed Pharmacother 2019, 112:108613. 
8. Zhou KI, Liu N, Pan T: Identification of N(6)-methyladenosine reader proteins. Methods 2017, 126:105-111.

9. Chen XY, Liang R, Yi YC, Fan HN, Chen M, Zhang J, Zhu JS: The m(6)A Reader YTHDF1 Facilitates the Tumorigenesis and Metastasis of Gastric Cancer via USP14 Translation in an m(6)A-Dependent Manner. Front Cell Dev Biol 2021, 9:647702.

10. Jing JJ, Zhao X, Li H, Sun LP, Yuan Y: Expression profiles and prognostic roles of m6A writers, erasers and readers in gastric cancer. Future Oncol 2021, 17(20):2605-2620.

11. Shen X, Zhao K, Xu L, Cheng G, Zhu J, Gan L, Wu Y, Zhuang Z: YTHDF2 Inhibits Gastric Cancer Cell Growth by Regulating FOXC2 Signaling Pathway. Front Genet 2020, 11:592042.

12. Liu T, Yang S, Cheng YP, Kong XL, Du DD, Wang X, Bai YF, Yin LH, Pu YP, Liang GY: The N6Methyladenosine (m6A) Methylation Gene YTHDF1 Reveals a Potential Diagnostic Role for Gastric Cancer. Cancer Manag Res 2020, 12:11953-11964.

13. Du D, Shen X, Zhang Y, Yin L, Pu Y, Liang G: Expression of long non-coding RNA SFTA1P and its function in non-small cell lung cancer. Pathol Res Pract 2020, 216(9):153049.

14. Lobo J, Barros-Silva D, Henrique R, Jerónimo C: The Emerging Role of Epitranscriptomics in Cancer: Focus on Urological Tumors. Genes (Basel) 2018, 9(11).

15. Pi J, Wang W, Ji M, Wang X, Wei X, Jin J, Liu T, Qiang J, Qi Z, Li F et al: YTHDF1 Promotes Gastric Carcinogenesis by Controlling Translation of FZD7. Cancer Res 2021, 81(10):2651-2665.

16. Lee D, Yu EJ, Ham IH, Hur H: Clinicopathological Implication of Insulin-like Growth Factor-II mRNABinding Protein 3 (IMP3) Expression in Gastric Cancer. Anticancer Res 2017, 37(1):135-142.

17. Wang X, Guan D, Wang D, Liu H, Wu Y, Gong W, Du M, Chu H, Qian J, Zhang Z: Genetic variants in m(6)A regulators are associated with gastric cancer risk. Arch Toxicol 2021, 95(3):1081-1088.

18. Yang $C$, Gong A: Integrated bioinformatics analysis for differentially expressed genes and signaling pathways identification in gastric cancer. Int J Med Sci 2021, 18(3):792-800.

19. Mancarella C, Scotlandi K: IGF2BP3 From Physiology to Cancer: Novel Discoveries, Unsolved Issues, and Future Perspectives. Front Cell Dev Biol 2019, 7:363.

20. Kim HY, Ha Thi HT, Hong S: IMP2 and IMP3 cooperate to promote the metastasis of triple-negative breast cancer through destabilization of progesterone receptor. Cancer Lett 2018, 415:30-39.

21. Liu H, Zeng Z, Afsharpad M, Lin C, Wang S, Yang H, Liu S, Kelemen LE, Xu W, Ma W et al: Overexpression of IGF2BP3 as a Potential Oncogene in Ovarian Clear Cell Carcinoma. Front Oncol 2019, 9:1570.

22. Mäkinen A, Nikkilä A, Haapaniemi T, Oksa L, Mehtonen J, Vänskä $M$, Heinäniemi M, Paavonen T, Lohi O: IGF2BP3 Associates with Proliferative Phenotype and Prognostic Features in B-Cell Acute Lymphoblastic Leukemia. Cancers (Basel) 2021, 13(7).

23. Gu Y, Niu S, Wang Y, Duan L, Pan Y, Tong Z, Zhang X, Yang Z, Peng B, Wang X et al: DMDRMRMediated Regulation of m(6)A-Modified CDK4 by m(6)A Reader IGF2BP3 Drives ccRCC Progression. Cancer Res 2021, 81(4):923-934. 
24. Zhang J, Ding F, Jiao D, Li Q, Ma H: The Aberrant Expression of MicroRNA-125a-5p/IGF2BP3 Axis in Advanced Gastric Cancer and Its Clinical Relevance. Technol Cancer Res Treat 2020, 19:1533033820917332.

25. Zhou Y, Huang T, Siu HL, Wong CC, Dong Y, Wu F, Zhang B, Wu WK, Cheng AS, Yu J et al: IGF2BP3 functions as a potential oncogene and is a crucial target of miR-34a in gastric carcinogenesis. $\mathrm{Mol}$ Cancer 2017, 16(1):77.

26. Yang Z, Jiang X, Zhang Z, Zhao Z, Xing W, Liu Y, Jiang X, Zhao H: HDAC3-dependent transcriptional repression of FOXA2 regulates $\mathrm{FTO} / \mathrm{m} 6 \mathrm{~A} / \mathrm{MYC}$ signaling to contribute to the development of gastric cancer. Cancer Gene Ther 2021, 28(1-2):141-155.

27. Yang Z, Jiang X, Li D, Jiang X: HBXIP promotes gastric cancer via METTL3-mediated MYC mRNA m6A modification. Aging (Albany NY) 2020, 12(24):24967-24982.

28. Yang DD, Chen ZH, Yu K, Lu JH, Wu QN, Wang Y, Ju HQ, Xu RH, Liu ZX, Zeng ZL: METTL3 Promotes the Progression of Gastric Cancer via Targeting the MYC Pathway. Front Oncol 2020, 10:115.

29. Anauate AC, Leal MF, Calcagno DQ, Gigek CO, Karia BTR, Wisnieski F, Dos Santos LC, Chen ES, Burbano RR, Smith MAC: The Complex Network between MYC Oncogene and microRNAs in Gastric Cancer: An Overview. Int J Mol Sci 2020, 21(5).

30. Cai MZ, Wen SY, Wang XJ, Liu Y, Liang H: MYC Regulates PHF8, Which Promotes the Progression of Gastric Cancer by Suppressing miR-22-3p. Technol Cancer Res Treat 2020, 19:1533033820967472.

31. Maués J, Ribeiro HF, Pinto GR, Lopes LO, Lamarão LM, Pessoa CMF, Moreira-Nunes CFA, de Carvalho RM, Assumpção PP, Rey JA et al: Gastric Cancer Cell Lines Have Different MYC-Regulated Expression Patterns but Share a Common Core of Altered Genes. Can J Gastroenterol Hepatol 2018, 2018:5804376.

32. Huang $\mathrm{H}$, Weng $\mathrm{H}$, Sun W, Qin X, Shi H, Wu H, Zhao BS, Mesquita A, Liu C, Yuan CL et al: Recognition of RNA N(6)-methyladenosine by IGF2BP proteins enhances mRNA stability and translation. Nat Cell Biol 2018, 20(3):285-295.

33. Chen G, Yang Z, Feng M, Wang Z: microRNA-217 suppressed epithelial-to-mesenchymal transition through targeting PTPN14 in gastric cancer. Biosci Rep 2020, 40(1).

34. Huang L, Wu RL, Xu AM: Epithelial-mesenchymal transition in gastric cancer. Am J Trans/ Res 2015, 7(11):2141-2158.

35. Yue B, Song C, Yang L, Cui R, Cheng X, Zhang Z, Zhao G: METTL3-mediated N6-methyladenosine modification is critical for epithelial-mesenchymal transition and metastasis of gastric cancer. $\mathrm{Mol}$ Cancer 2019, 18(1):142.

36. Cheng C, Wu Y, Xiao T, Xue J, Sun J, Xia H, Ma H, Lu L, Li J, Shi A et al: METTL3-mediated m(6)A modification of ZBTB4 mRNA is involved in the smoking-induced EMT in cancer of the lung. $\mathrm{Mol}$ Ther Nucleic Acids 2021, 23:487-500.

37. Su P, Hu J, Zhang H, Li W, Jia M, Zhang X, Wu X, Cheng H, Xiang L, Zhou G: IMP3 expression is associated with epithelial-mesenchymal transition in breast cancer. Int J Clin Exp Pathol2014, 7(6):3008-3017. 
38. Meškytė EM, Keskas S, Ciribilli Y: MYC as a Multifaceted Regulator of Tumor Microenvironment Leading to Metastasis. Int J Mol Sci 2020, 21(20).

\section{Figures}
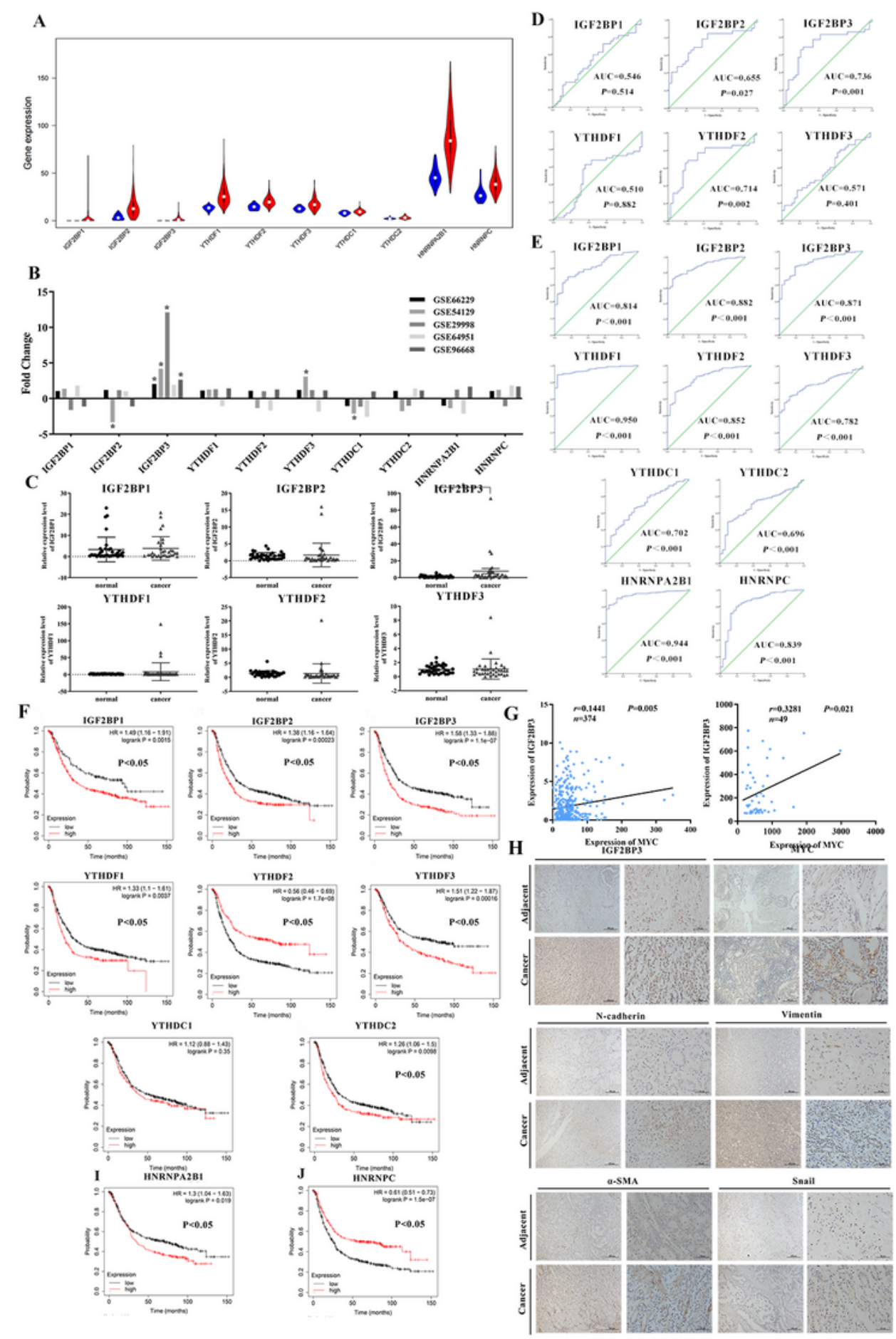

Figure 1 
Elevated IGF2BP3 expression was related to the diagnosis and the poor prognosis of GC patients. A The m6A readers expression in GC based on the TCGA databases, $* \mathbb{F C} \varangle>2$ and $P<0.05$. B The m6A readers expression in $\mathrm{GC}$ based on the GEO databases, $* \mathbb{F C} \nabla>2$ and $P<0.05$. C The m6A reader mRNA expression in 40 couples of gastric cancer and para-carcinoma tissues through the qRT-PCR experiment, ${ }^{\star} P<0.05$. For the above qRT-PCR data, relative quantification normalized to $\beta$-actin. D The ROC analysis of the diagnosis of m6A reader based on the human gastric cancer tissue. E The ROC analysis of the diagnose of the m6A reader based on the TCGA database. $F$ Kaplan-Meier testing revealed the relations between m6A reader expression and survival prognosis in GC patients based on the TCGA database. $\mathbf{G}$ The correlation between IGF2BP3 and MYC was verified from the TCGA database and the human gastric cancer tissue. $\mathbf{H}$ The expression of IGF2BP3, MYC and EMT markers ( $N$-cadherin, Vimentin, a-SMA and Snail) in the human gastric cancer tissue was measured by IHC, original magnification, $\times 100$ (top), $\times 400$ (bottom).

A
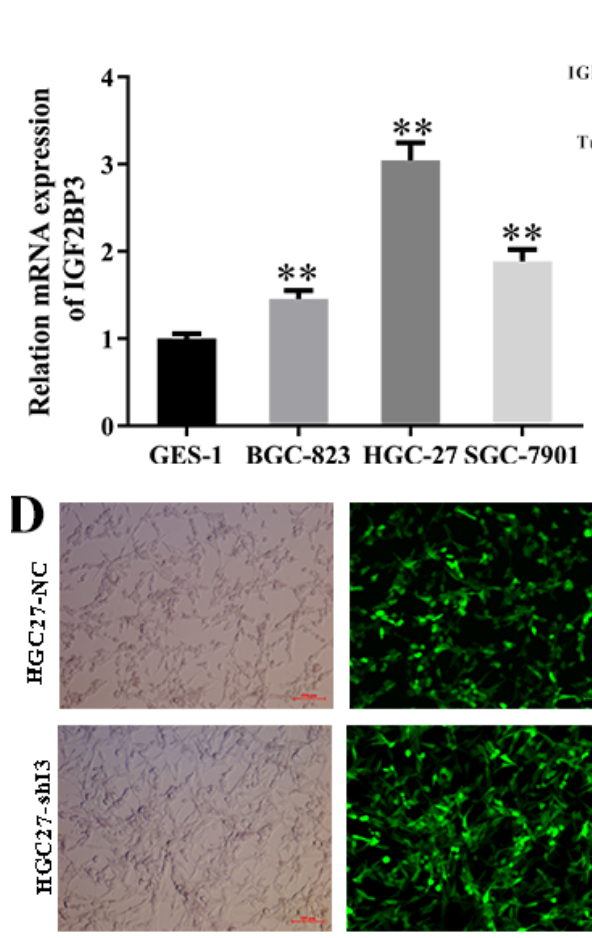

\section{$\mathbf{H}$}

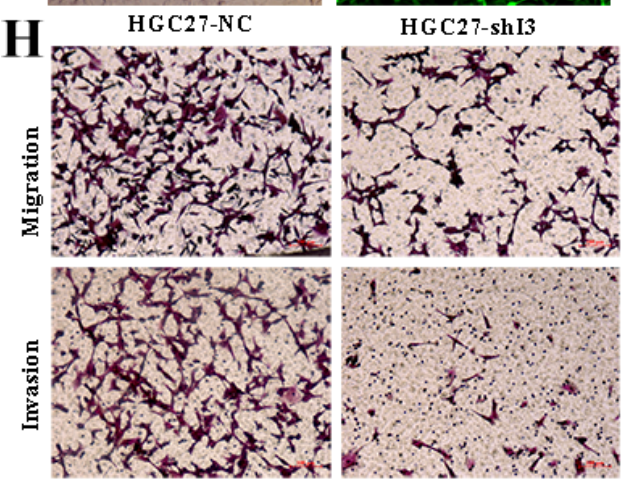

B

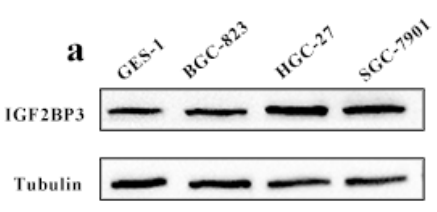

b
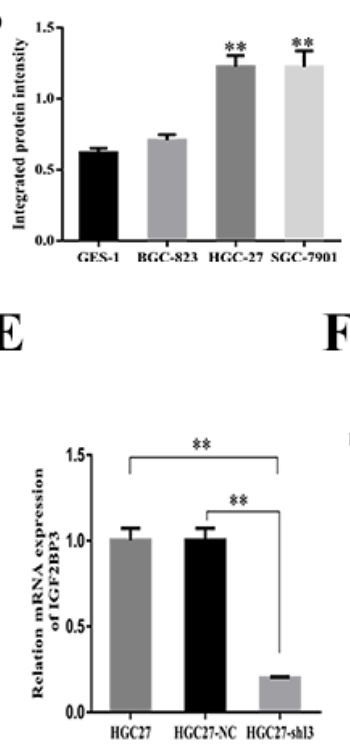

$\mathbf{E}$
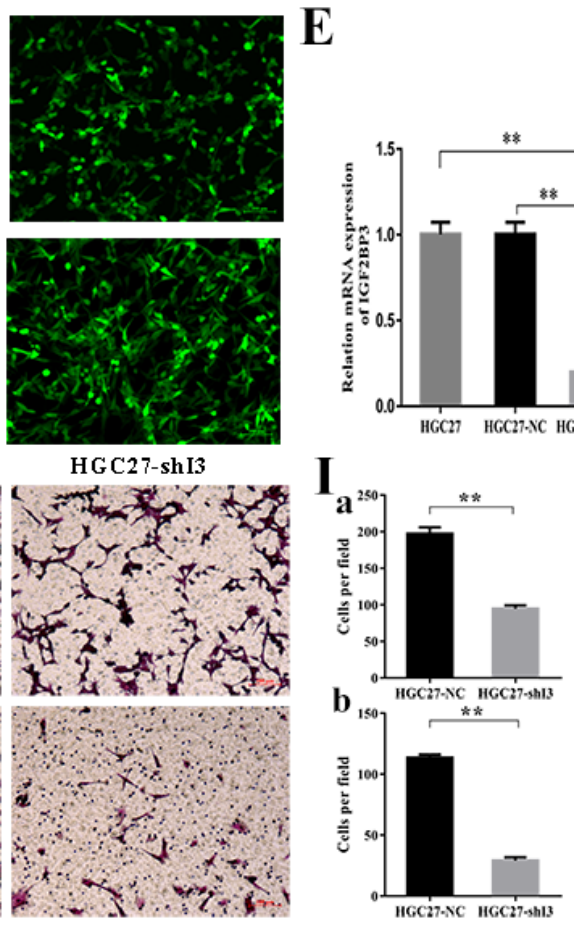

C

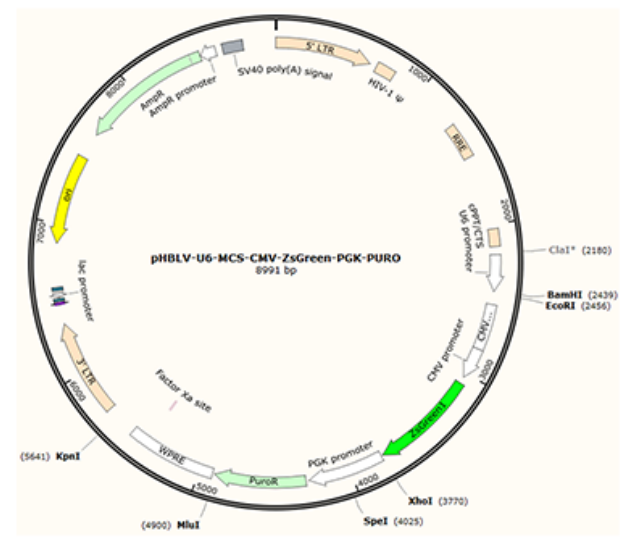

$\mathbf{F}$

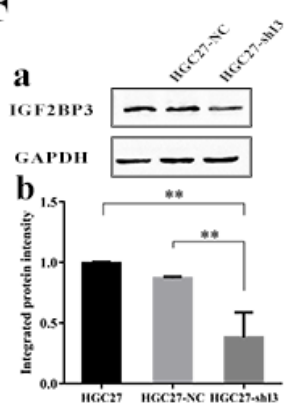

G

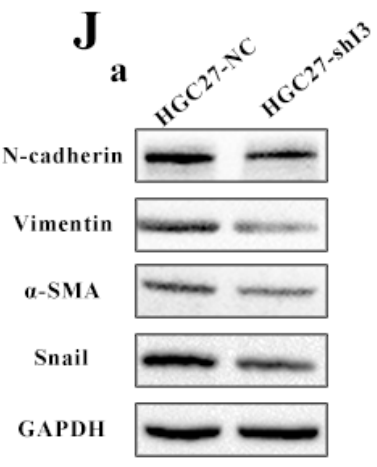

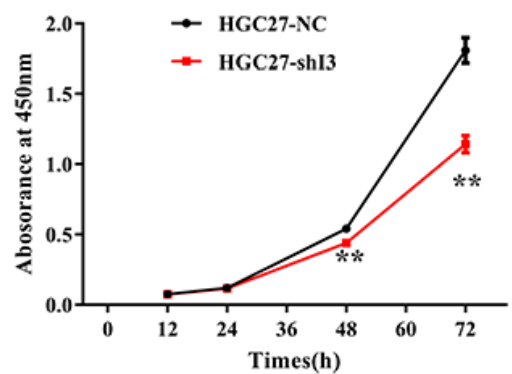

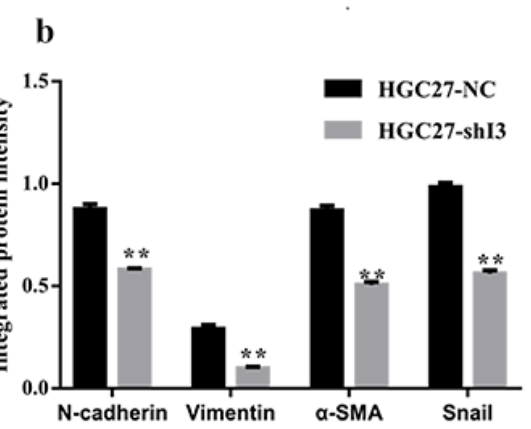


Figure 2

IGF2BP3 was up-regulated and promoted proliferation, invasion, migration, and EMT processes in GC. A The IGF2BP3 mRNA and protein levels were measured in GES-1, BGC-823, HGC-27 and SGC-7901 cells by using qRT-PCR experiment, ${ }^{*} P<0.01$ vs. GES-1. B The IGF2BP3 protein levels were measured in GES-1 and BGC-823, HGC-27, SGC-7901 cells. a The protein expression was analyzed by WB. $\mathbf{b}$ The band densities of specific proteins were quantified by ImageJ software, ${ }^{\star} P<0.01$ vs. GES-1. C The diagram was the IGF2BP3 Knockdown lentivirus empty vector. D The transfection efficiency was detected after the HGC-27 GC cells were transfected with shNC and IGF2BP3 shRNA, namely Blank group (HGC27-shNC) and IGF2BP3 knockdown group (HGC27-shl3). E The IGF2BP3 mRNA levels were measured after transfection with shIGF2BP3 and shNC in HGC-27 cells by the qRT-PCR experiment, ${ }^{\star *} P<0.01$. F The IGF2BP3 protein levels and the relative grayscale value were measured after transfection with shIGF2BP3 and shNC in HGC-27 cells. a The protein expression was analyzed by WB. $\mathbf{b}$ The band densities of specific proteins were quantified by ImageJ software, ${ }^{\star *} \mathrm{P}<0.01$. $\mathbf{G}$ The cell proliferative capacity was evaluated by CCK-8 array. ${ }^{*} P<0.01$. H The transwell experiment displayed that IGF2BP3 knockdown repressed the HGC-27 GC cells migration and invasion. I The relative HGC-27 cells invasion and migration levels were measured in HGC27-shl3 cells and HGC27-shNC cells. a The relative cell migration levels, ${ }^{*} * P<0.01$. b The relative cell invasion levels, ${ }^{\star *} P<0.01$. J The EMT markers ( $\mathrm{N}$-cadherin, Vimentin, a-SMA and Snail) protein expression was measured in HGC27-shl3 cells and HGC27-shNC cells. a The protein expression was analyzed by WB. $\mathbf{b}$ The band densities of specific proteins were quantified by ImageJ software, $* \star P<0.01$.
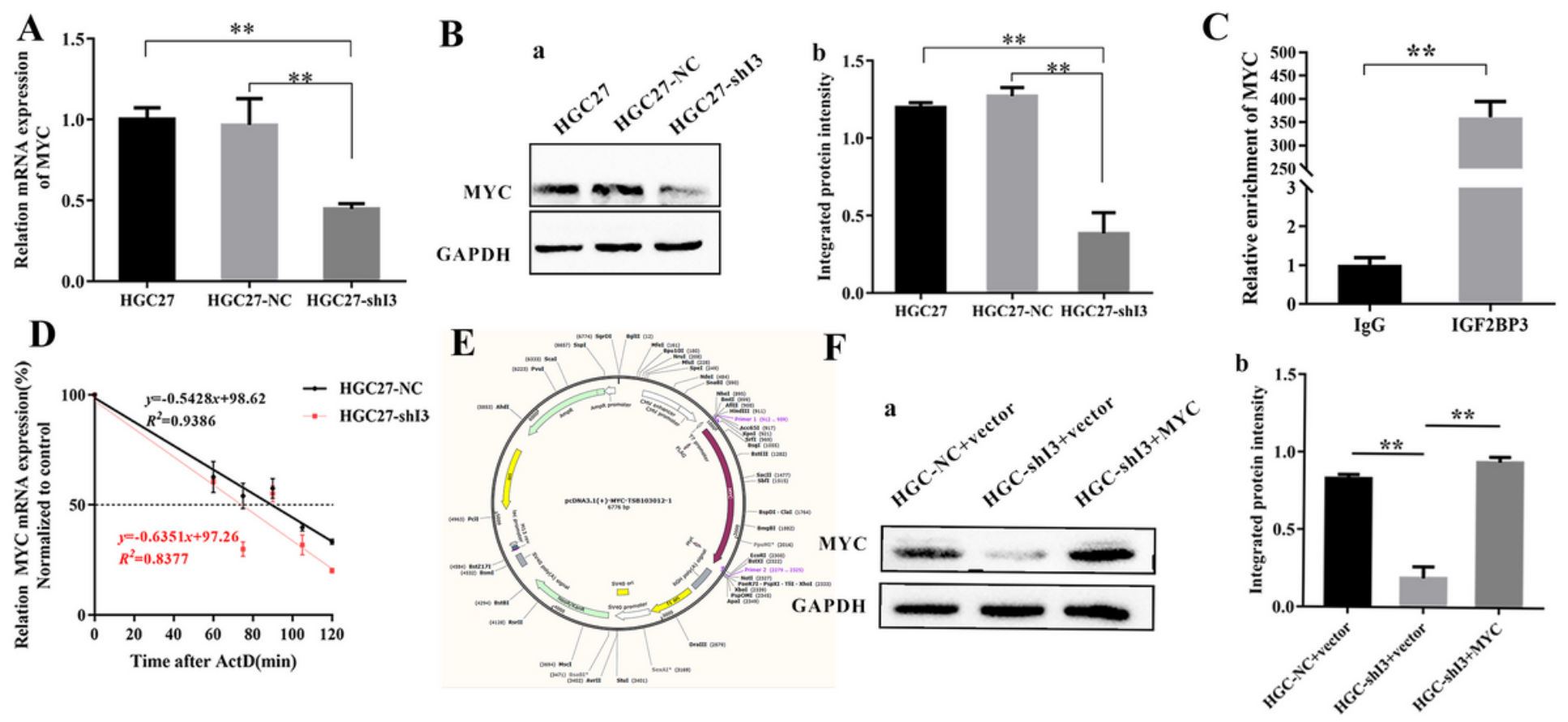

Figure 3

IGF2BP3 can combine with MYC mRNA to enhance MYC mRNA stability and the expression. A The MYC mRNA expression level in HGC27-shl3 cells and HGC27-shNC cells was measured by the qRT-PCR 
experiment, ${ }^{*} P<0.01$. B The MYC protein expression level was evaluated in HGC27-shl3 cells and HGC27shNC cells. $\mathbf{a}$ The protein expression was analyzed by WB. $\mathbf{b}$ The band densities of specific proteins were quantified by ImageJ software, ${ }^{*} P<0.01$. C The MYC mRNA was abundant in IGF2BP3 RIP of HGC-27 compared with IgG RIP was verified by RIP-qRT-PCR experiment, ${ }^{\star} P<0.01$ vs. IgG. D The MYC transcripts stability in ActD-treated cells after transfected with MYC overexpression plasmids in HGC-27 cells was displayed by the qRT-PCR experiment, $* * P<0.01$. E The diagram was the overexpression MYC knockdown plasmids empty vector. $\mathbf{F}$ The MYC protein levels and relative grayscale value expression after transfected with MYC overexpression plasmids in HGC-27 cells was detected. a The protein expression was measured by WB. b The band densities of specific proteins were quantified by ImageJ software, $\star \star P<0.01$. 
A

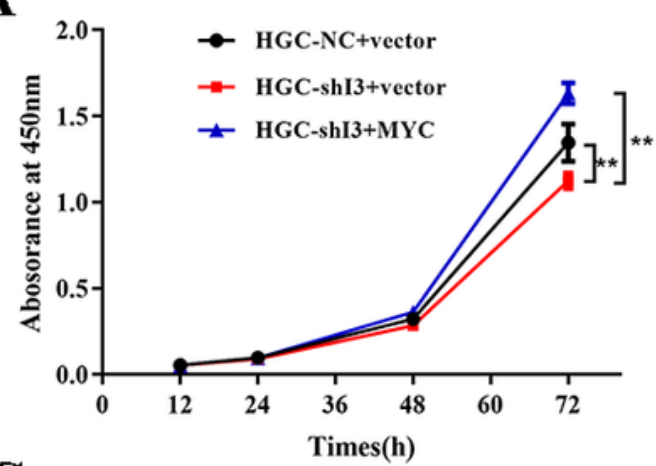

B

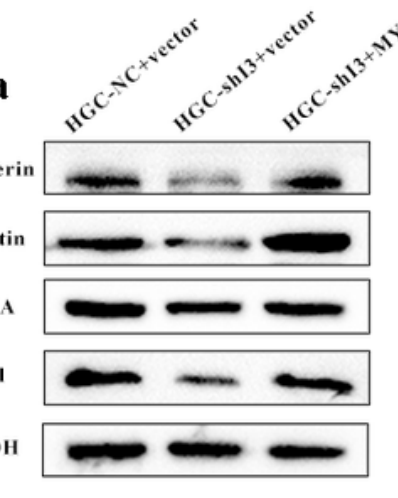

GAPDH HGC-sh I3+vector

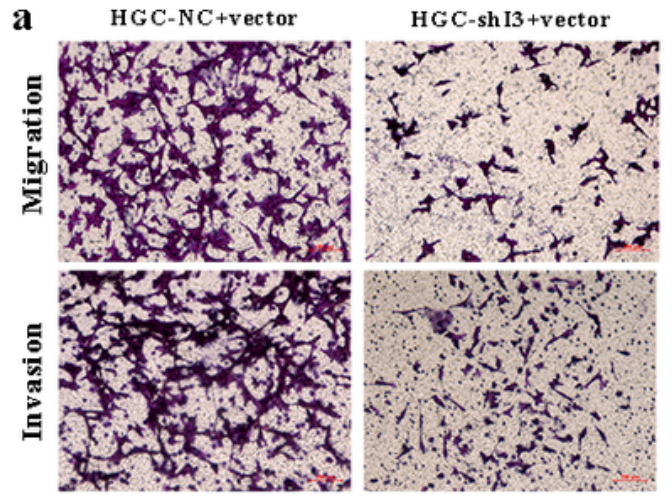
HGC-shI3+MYC
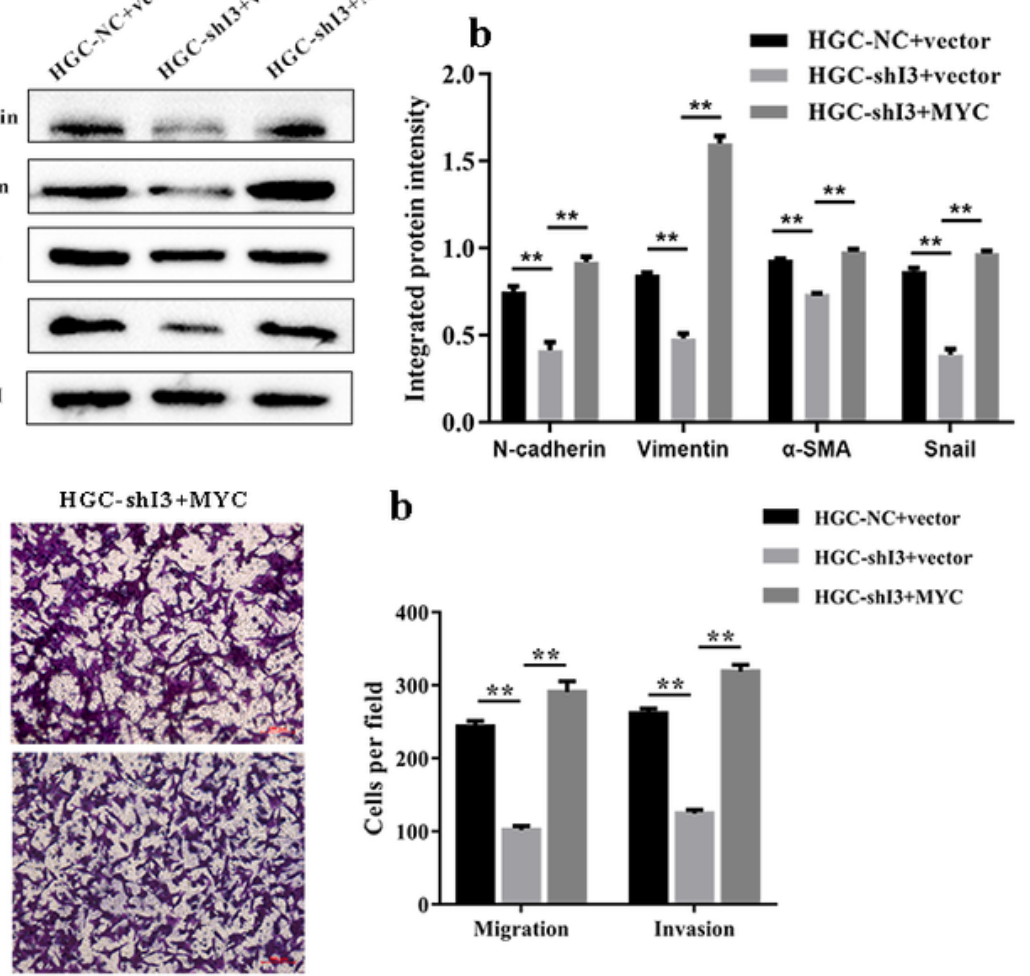

b

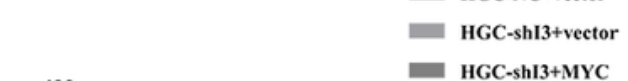

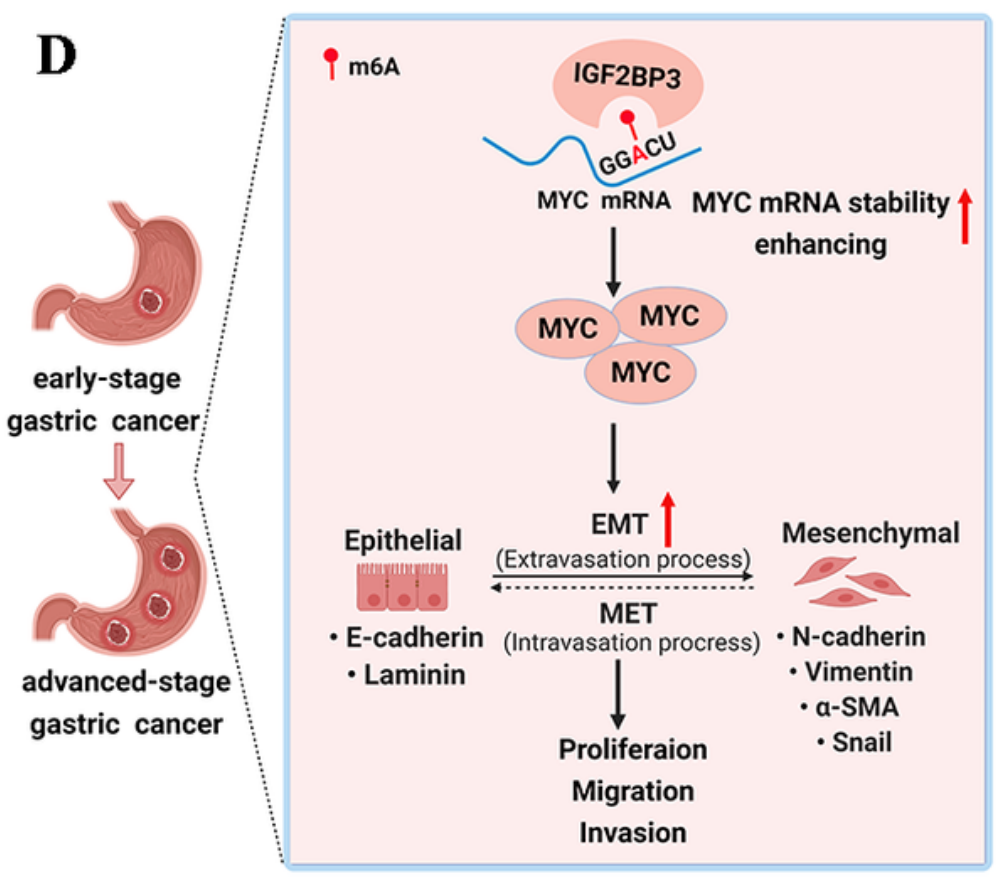

Figure 4

MYC overexpression effectively reverses the inhibition of IGF2BP3-knockdown-induced GC cells tumor progression. A The GC cells proliferation ability was measured by CCK8 experiment, ${ }^{\star \star} P<0.01$. B The EMT markers ( $\mathrm{N}$-cadherin, Vimentin, a-SMA and Snail) protein expression level of HGC-27-shl3 cells after transfected with MYC overexpression plasmid were evaluated. a The protein expression was measured by WB. $\mathbf{b}$ The band densities of specific proteins were quantified by ImageJ software, ${ }^{\star *} P<0.01$. C The HGC- 
27-shl3 cells migration and invasion ability after transfection with MYC overexpression plasmids were elucidated. a The transwell assay results. $\mathbf{b}$ The relative cell migration and invasion levels, $* \star P<0.01$. D The illustration summarizes the function and mechanism of the IGF2BP3-MYC axis in gastric cancer. 